\title{
Chasing Convex Bodies with Linear Competitive Ratio
}

\author{
C. J. ARGUE, ANUPAM GUPTA, and ZIYE TANG, Carnegie Mellon University \\ GURU GURUGANESH, Google Research
}

We study the problem of chasing convex bodies online: given a sequence of convex bodies $K_{t} \subseteq \mathbb{R}^{d}$ the algorithm must respond with points $x_{t} \in K_{t}$ in an online fashion (i.e., $x_{t}$ is chosen before $K_{t+1}$ is revealed). The objective is to minimize the sum of distances between successive points in this sequence. Bubeck et al. (STOC 2019) gave a $2^{O(d)}$-competitive algorithm for this problem. We give an algorithm that is $O(\min (d, \sqrt{d \log T}))$ competitive for any sequence of length $T$.

CCS Concepts: • Theory of computation $\rightarrow$ Online algorithms; Convex optimization; Computational geometry;

Additional Key Words and Phrases: Online algorithms, convex body chasing, Steiner point, work function

\section{ACM Reference format:}

C. J. Argue, Anupam Gupta, Ziye Tang, and Guru Guruganesh. 2021. Chasing Convex Bodies with Linear Competitive Ratio. F. ACM 68, 5, Article 32 (August 2021), 10 pages.

https://doi.org/10.1145/3450349

\section{INTRODUCTION}

In the problem of chasing convex bodies, we control a point in $\mathbb{R}^{d}$, initially at $x_{0}=0$. At each timestep $t$, the adversary gives a closed convex set $K_{t} \subseteq \mathbb{R}^{d}$ as its request, and we must respond with a point $x_{t} \in K_{t}$. (In essence, we are moving our point to lie within $K_{t}$.) This response must be done in an online fashion, namely, $x_{t}$ must be chosen before the next set $K_{t+1}$ is revealed. The goal is to minimize the total movement cost: if the process goes on for $T$ steps, this cost is

$$
A L G:=\sum_{t=1}^{T}\left\|x_{t}-x_{t-1}\right\| .
$$

We focus on the Euclidean norm; there are other results known for general norms (see, e.g., [8] for results and references). We work in the framework of competitive analysis; we compare the algorithm's cost to the optimal cost OPT incurred by an all-knowing adversary, and the ratio between the two is called the competitive ratio.

Friedman and Linial [11] introduced the problem of chasing convex bodies as a generalization of several existing online problems. They showed that no algorithm can achieve a competitive ratio

Anupam Gupta was supported in part by NSF award CCF-1907820.

Authors' addresses: C. J. Argue, A. Gupta, and Z. Tang, Carnegie Mellon University, 5000 Forbes Avenue, Pittsburgh, PA 15217; emails: cargue@andrew.cmu.edu, anupamg@cs.cmu.edu, ziyet@andrew.cmu.edu; G. Guruganesh, Google Research, 1600 Amphitheatre Pkwy, Mountain View, CA 94043; email: gurug@google.com.

Permission to make digital or hard copies of all or part of this work for personal or classroom use is granted without fee provided that copies are not made or distributed for profit or commercial advantage and that copies bear this notice and the full citation on the first page. Copyrights for components of this work owned by others than the author(s) must be honored. Abstracting with credit is permitted. To copy otherwise, or republish, to post on servers or to redistribute to lists, requires prior specific permission and/or a fee. Request permissions from permissions@acm.org.

(C) 2021 Copyright held by the owner/author(s). Publication rights licensed to ACM.

0004-5411/2021/08-ART32 \$15.00

https://doi.org/10.1145/3450349

Journal of the ACM, Vol. 68, No. 5, Article 32. Publication date: August 2021. 
lower than $\sqrt{d}$, gave an algorithm for $d=2$, and posed getting an $O(f(d))$-competitive algorithm for general $d$ as an open problem. Over the years, competitive algorithms were given for special cases (see, e.g., $[2,11,12,18])$. Motivated by problems in data center scheduling, this problem was also studied under the name of smoothed online convex optimization (SOCO) (see, e.g., [10, 13]).

A particularly relevant line of recent work is that for nested convex body chasing, where the request sequence satisfies $K_{t} \supseteq K_{t+1}$ for all $t$. For this case, a series of works $[3,4]$ culminated in Bubeck et al. [8] giving an $O(\min (d, \sqrt{d \log T}))$-competitive algorithm based on the classical notion of the Steiner point of convex bodies, and a more sophisticated algorithm with a nearoptimal competitive ratio of $O(\sqrt{d \log d})$. Moreover, a parallel paper of Bubeck et al. [9] built on the recursive centroid approach from [3] to give the first $f(d)$-competitive algorithm for the general non-nested case in arbitrary dimension $d$, thereby resolving the open problem from [11]. Their algorithm has competitive ratio $f(d)=2^{O(d)}$, and it substantially extends the recursive approach of [3] for the nested case using a several new arguments. Our main result gives a simpler algorithm with an improved competitive ratio.

THEOREM 1.1. There is an $O(\min (d, \sqrt{d \log T}))$-competitive algorithm for the general convex body chasing problem in d-dimensional Euclidean space.

Our algorithm appears in Section 2. It uses the Steiner-point based algorithm for the nested case [8] applied in a black-box fashion to a suitable body $\Omega_{t}$. The body is defined using the concept of work functions commonly used in online algorithms (see, e.g., [7, 14, 18]). Hence, our result relies on bringing together the two lines of investigation that had held out hope for the problem: ideas based on the work function, and those based on convex geometry. In Section 3, we give the details of an efficient implementation. The reader not interested in these computational issues can focus only on Section 2.

Friedman and Linial also defined a more general problem of convex function chasing, as a direct generalization of metrical task systems [7]. Here, the request at time $t$ is a convex function $g_{t}: \mathbb{R}^{d} \rightarrow \mathbb{R}_{\geq 0}$, and the algorithm must again respond with a point $x_{t} \in \mathbb{R}^{d}$. The goal is to minimize $\sum_{t}\left(g_{t}\left(x_{t}\right)+\left\|x_{t}-x_{t-1}\right\|\right)$, i.e., the total hit cost in addition to the movement cost. Setting $g_{t}$ to be the indicator of a convex set $K_{t}$ gives us back the problem of chasing convex bodies. Bubeck et al. [9] showed that convex function chasing in $d$ dimensions can be reduced to convex body chasing in $d+1$ dimensions using the epigraph. This reduction loses only a constant factor in the competitive ratio. Hence, we get $O(\min (d, \sqrt{d \log T}))$-competitiveness for chasing convex functions as well. Independent and concurrent work by M. Sellke extends the notion of Steiner points to functional Steiner points for convex functions in order to give a $\min (d, O(\sqrt{d \log T}))$-competitive ratio for convex function chasing, and hence for convex bodies as well [17]. His results hold for more general norms.

\subsection{Notation}

Given an instance of convex body chasing, the work function $w_{t}(x)$ at time $t$ for the point $x \in \mathbb{R}^{d}$ is the cost of the optimal trajectory that starts at $x_{0}$, satisfies the first $t$ requests, and ends at the point $x$. Formally, it is given by the convex program:

$$
\begin{aligned}
w_{t}(x)=\min & \sum_{s=1}^{t}\left\|x_{s}-x_{s-1}\right\|+\left\|x_{t}-x\right\| \\
\text { s.t. } & x_{t} \in K_{t} \\
& x_{0}=0 .
\end{aligned}
$$




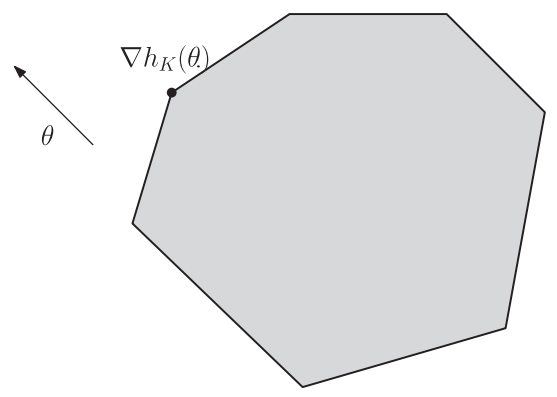

Fig. 1. $\nabla h_{K}(\theta)$ is the maximizer of $\max _{x \in K}\langle\theta, x\rangle$.

Let $K \subseteq \mathbb{R}^{d}$ be a bounded convex body, and let $\operatorname{cg}(K)=\frac{\int_{x \in K} x d x}{\int_{x \in K} d x}$ denote the center of gravity of $K$. (This is also called the center of mass, the centroid, or the barycenter of the body.) Let $B\left(x_{0}, R\right):=$ $\left\{x \in \mathbb{R}^{d}:\left\|x-x_{0}\right\| \leq R\right\}$ denote the ball of radius $R$ centered at $x_{0}$, and let $B:=B(0,1)$ denote the unit ball in $\mathbb{R}^{d}$. The Steiner point of the convex body $K$ is [15]

$$
\operatorname{st}(K):=\lim _{s \rightarrow \infty} \operatorname{cg}(K+s B),
$$

where the sum is the Minkowski sum $K_{1}+K_{2}:=\left\{x_{1}+x_{2} \mid x_{i} \in K_{i}\right\}$. The Steiner point has several other equivalent characterizations, including these two [16, Section 5.4]:

$$
\begin{aligned}
& \operatorname{st}(K)=d \int_{\theta \in S^{d-1}} \theta h_{K}(\theta) d \omega(\theta)=d \mathbb{E}_{\theta \sim \omega}\left[\theta h_{K}(\theta)\right] \text { and } \\
& \operatorname{st}(K)=\int_{\theta \in S^{d-1}} \nabla h_{K}(\theta) d \omega(\theta)=\mathbb{E}_{\theta \sim \omega}\left[\nabla h_{K}(\theta)\right],
\end{aligned}
$$

where $\omega$ is the uniform (isometry invariant) measure on $S^{d-1}$ with $\omega\left(S^{d-1}\right)=1$, the function

$$
h_{K}(\theta):=\max _{x \in K}\langle\theta, x\rangle
$$

is the support function of the convex body $K$, and its gradient

$$
\nabla h_{K}(\theta):=\arg \max _{x \in K}\langle\theta, x\rangle
$$

is the maximizing point (see Figure 1 for an example). We will use definitions (1) and (2) in the two proofs for the algorithm, and Equation (2) for the efficient implementation. Definition (3) shows that $\operatorname{st}(K) \in K$, which is crucial to the proof of Bubeck et al. in [8], though we will not need this property for our proofs. See, e.g., $[6,16]$, for more about the history and properties of the Steiner point.

\section{THE ALGORITHM}

The algorithm has an outer guess-and-double step, where we maintain a current estimate $r$ that lies in $[O P T / 2, O P T]$. Given such an $r$, the algorithm is a single sentence: at each time $t$, we take the $2 r$-level set $\Omega_{t}$ of the work function $w_{t}(x)$, and move to its Steiner point.

LEMMA 2.1. The work function $w_{t}$ is a convex function, and $\Omega_{t}$ is a bounded convex set.

Proof. For $x, y \in \mathbb{R}^{d}$, let $\left\{x_{s}\right\}_{s=1}^{t},\left\{y_{s}\right\}_{s=1}^{t}$ be the optimal solutions to the convex program that witness the values $w_{t}(x), w_{t}(y)$. Consider $z:=\lambda x+(1-\lambda) y$. Then, for each timestep $s \leq t$, define 


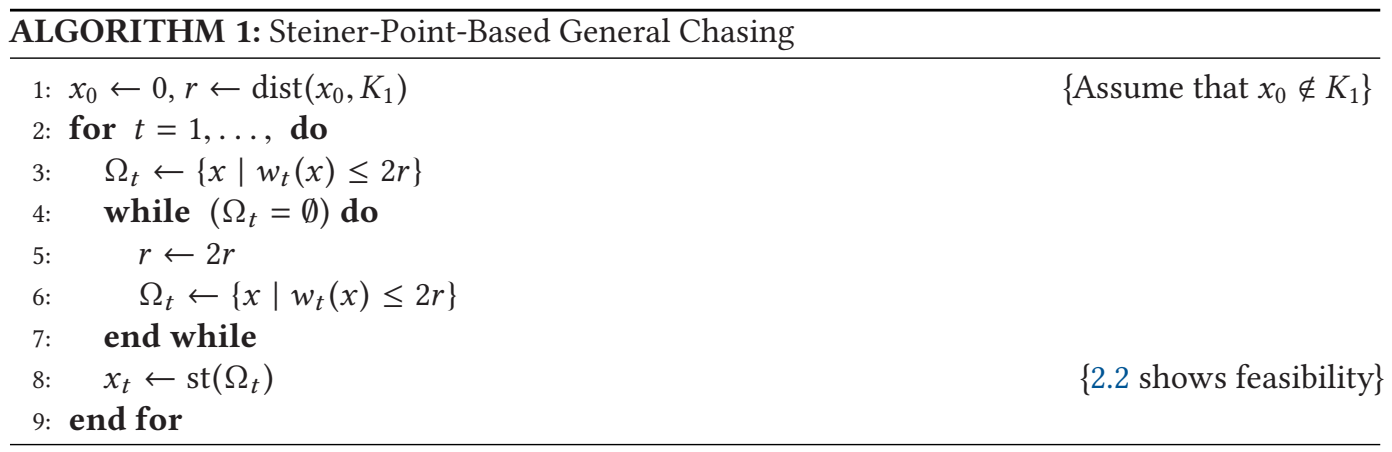

$z_{s}:=\lambda x_{s}+(1-\lambda) y_{s}$ and note that $z_{s} \in K_{s}$ by convexity. Therefore,

$$
w_{t}(z) \leq \sum_{s=1}^{t}\left\|z_{s}-z_{s-1}\right\|+\left\|z_{t}-z\right\| \leq \lambda w_{t}(x)+(1-\lambda) w_{t}(y),
$$

where the first inequality is because $\left\{z_{s}\right\}$ is a feasible solution to the convex program for point $z$ and $w_{t}(z)$ is the minimum value for it, and the second inequality is by the convexity of the norm. Hence, $w_{t}(\cdot)$ is a convex function, and its (sub-)level sets $\Omega_{t}$ are convex sets. Moreover, $\Omega_{t} \subseteq\{x \mid\|x\| \leq 2 r\}$ and hence is bounded.

The all-important next claim shows that our choice of $x_{t}$ in Step 8 is feasible.

Lemma 2.2. With $\Omega_{t}$ and $x_{t}$ as defined in the algorithm, $x_{t} \in K_{t}$.

Proof. By the first definition (1) of $\operatorname{st}\left(\Omega_{t}\right)$, it suffices to show that for any fixed $s \geq 0$ we have $\operatorname{cg}\left(\Omega_{t}+s B\right) \in K_{t}$. To this end, we show that $\operatorname{cg}\left(\Omega_{t}+s B\right)$ is contained in every halfspace containing $K_{t}$.

Let $H:=\left\{x \in \mathbb{R}^{d} \mid\langle a, x\rangle \geq b\right\}$ be a halfspace containing $K_{t}$ and let $H^{=}:=\left\{x \in \mathbb{R}^{d} \mid\langle a, x\rangle=b\right\}$ denote the supporting hyperplane of this halfspace. (Assume that $a$ is a unit vector.) For $x \notin H$, define $\rho(x)$ to be the reflection of $x$ across $H^{=}$:

$$
\rho(x):=x+2(b-\langle a, x\rangle) a .
$$

Consider that

$$
w_{t}(x)=\min _{y \in K_{t}}\left\{\|x-y\|+w_{t-1}(y)\right\}
$$

Let $y$ be the argmin of the expression on the right. Using that fact that $y \in K_{t} \subseteq H$, we have

$$
w_{t}(x)=\|x-y\|+w_{t-1}(y) \geq\|\rho(x)-y\|+w_{t-1}(y) \geq w_{t}(\rho(x)) .
$$

It follows that if the point $x \in \Omega_{t} \backslash H$, then its reflection $\rho(x) \in \Omega_{t}$ as well.

A similar argument holds for any point $z \in\left(\Omega_{t}+s B\right) \backslash H$ : we claim that $\rho(z) \in \Omega_{t}+s B$. Get $x \in \Omega_{t}$ with $\|z-x\| \leq s$. If $x \in H$, then $\|\rho(z)-x\| \leq\|z-x\|$ so $\rho(z) \in \Omega_{t}+s B$. Suppose $x \notin H$. The above argument shows that $\rho(x) \in \Omega_{t}$ and furthermore $\|\rho(z)-\rho(x)\|=\|z-x\| \leq s$, so $\rho(z) \in \Omega_{t}+s B$. Hence, the reflection of the part of $\Omega_{t}+s B$ that is infeasible for $H$ actually lies within $\Omega_{t}+s B$ : namely, for any $s \geq 0$,

$$
\rho\left(\left(\Omega_{t}+s B\right) \backslash H\right) \subseteq\left(\Omega_{t}+s B\right) \cap H .
$$

For convenience, we will write the convex body $\Omega_{t}+s B=\Omega^{-} \cup \Omega^{+} \cup \widehat{\Omega}$ (see Figure 2) where

$$
\begin{aligned}
& -\Omega^{-}:=\left(\Omega_{t}+s B\right) \backslash H . \\
& -\Omega^{+}:=\rho\left(\Omega^{-}\right) . \\
& -\widehat{\Omega}:=\left[\left(\Omega_{t}+s B\right) \cap H\right] \backslash \Omega^{+} .
\end{aligned}
$$



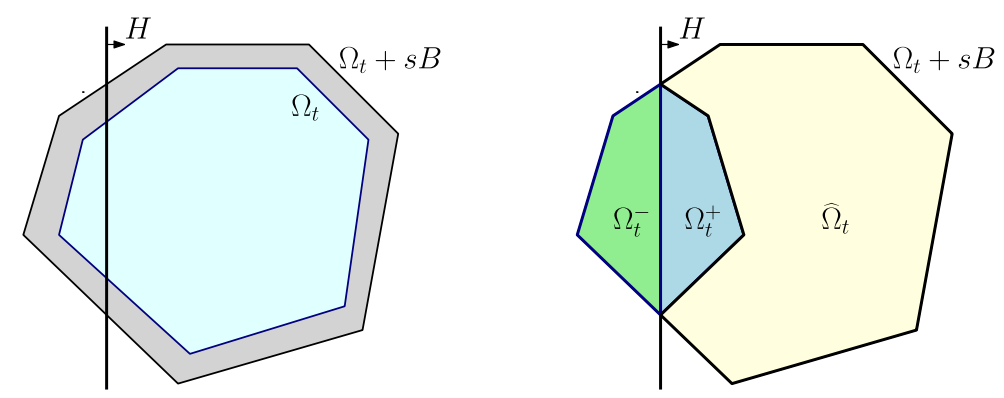

Fig. 2. Proof of Lemma 2.2.

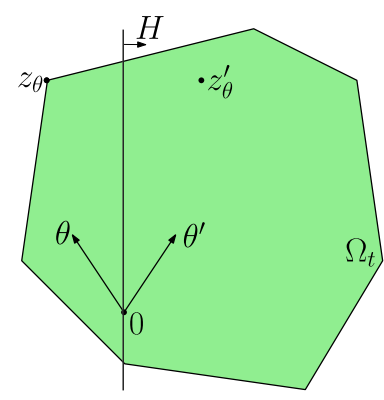

Fig. 3. Alternate proof of Lemma 2.2.

By symmetry, $\operatorname{cg}\left(\Omega^{-} \cup \Omega^{+}\right)$lies on $H^{=}$. Since $\widehat{\Omega} \subseteq H$, we have $\operatorname{cg}(\widehat{\Omega}) \in H$. Letting $\gamma=\frac{\operatorname{Vol}\left(\Omega^{+} \cup \Omega^{-}\right)}{\operatorname{Vol}\left(\Omega_{t}+s B\right)}$, it follows that

$$
\operatorname{cg}\left(\Omega_{t}+s B\right)=\operatorname{cg}\left(\left(\Omega^{-} \cup \Omega^{+}\right) \cup \widehat{\Omega}\right)=\gamma \cdot \operatorname{cg}\left(\Omega^{-} \cup \Omega^{+}\right)+(1-\gamma) \cdot \operatorname{cg}(\widehat{\Omega}) \in H .
$$

But $H$ was chosen to be a generic halfspace containing $K_{t}$, so $\operatorname{cg}\left(\Omega_{t}+s B\right)$ is contained in every halfspace $H$ containing $K_{t}$.

An Alternate Proof of 2.2. This proof follows a reflection argument similar to the one in the first proof, but is based on definition (2) of the Steiner point. By translating the frame of reference, assume that the origin lies in $H^{=}$, namely, $b=0$.

Consider some direction $\theta \in S^{d-1}$ such that $\langle\theta, a\rangle \leq 0$ (i.e., $\theta$ lies to the left of the separating hyperplane in Figure 3). Let $z_{\theta} \in \Omega_{t}$ be such that $h_{\Omega_{t}}(\theta)=\left\langle\theta, z_{\theta}\right\rangle$. If $z_{\theta} \in H$, then define $z_{\theta}^{\prime}=z_{\theta}$, else define $z_{\theta}^{\prime}=\rho\left(z_{\theta}\right)$ to be the reflection of $z_{\theta}$ across $H^{=}$. By the arguments above, $z_{\theta}^{\prime} \in \Omega_{t}$ as well, and moreover by construction $z_{\theta}^{\prime} \in H$. Finally, let $\theta^{\prime}:=\rho(\theta)$ be the reflection of $\theta$ across $H^{=}$. We claim that

$$
\left\langle z_{\theta}^{\prime}, \theta^{\prime}\right\rangle \geq\left\langle z_{\theta}, \theta\right\rangle
$$

Indeed, we can rewrite (5) as $\left\langle z_{\theta}^{\prime}-z_{\theta}, \theta^{\prime}\right\rangle+\left\langle z_{\theta}, \theta^{\prime}-\theta\right\rangle \geq 0$. If $z_{\theta} \in H$, then the first term is 0 and the second term is $2\left\langle z_{\theta}, a\right\rangle\left\langle\theta^{\prime}, a\right\rangle \geq 0$ (since $\theta$ is the reflection of $\theta^{\prime}$ across $H^{=}$). Otherwise, the first term is $-2\left\langle z_{\theta}, a\right\rangle\left\langle a, \theta^{\prime}\right\rangle$ and the second term is $2\left\langle z_{\theta}, a\right\rangle\left\langle\theta^{\prime}, a\right\rangle$, hence their sum is 0 . This proves Equation (5), and we can infer that

$$
h_{\Omega_{t}}\left(\theta^{\prime}\right) \geq\left\langle z_{\theta}^{\prime}, \theta^{\prime}\right\rangle \stackrel{(5)}{\geq}\left\langle z_{\theta}, \theta\right\rangle=h_{\Omega_{t}}(\theta)
$$


where the first inequality follows from the definition of the support function as the maximizer. So $\theta h_{\Omega_{t}}(\theta)+\theta^{\prime} h_{\Omega_{t}}\left(\theta^{\prime}\right)$ is a point in $H$. Averaging over the choices of $\theta$ gives us a point also in $H$. Since $H^{=}$contains the origin, scaling by $d$ still gives a point in $H$.

Theorem 1.1 is now proved by applying standard doubling arguments (as in [3]) to the results of [8].

Proof of Theorem 1.1. We consider the progression of the algorithm in phases; each new phase begins when $r$ changes. Suppose the phase corresponding to some value of $r$ consists of times $\left\{t_{1}, \ldots, t_{2}\right\}$. From the fact that the work function is non-decreasing over time, i.e., $w_{t} \leq w_{t+1}$, it follows that

$$
\Omega_{t_{1}} \supseteq \Omega_{t_{1}+1} \supseteq \cdots \supseteq \Omega_{t_{2}} .
$$

Moreover, $B(0,2 r) \supseteq \Omega_{t}$ for each $t$ in this phase. This gives an instance of the nested body chasing problem. From Bubeck et al. [8, Theorem 3.3], the algorithm that moves to the Steiner point $\operatorname{st}\left(\Omega_{t}\right)$ at each time pays at most $(2 r) \cdot O(\min (d, \sqrt{d \log T}))$. It follows that our algorithm pays at most that quantity plus an additive $O(r)$ during the phase, where the extra $O(r)$ comes from imaginarily moving to 0 at the beginning of the phase. Summing over all phases, we pay at most $r_{\text {final }} \cdot O(\min (d, \sqrt{d \log T}))$, whereas $O P T$ pays at least $r_{\text {final }}$. Hence the proof.

\section{AN EFFICIENT IMPLEMENTATION FOR CHASING HALFSPACES}

We now give an efficient implementation of our $O(\min (d, \sqrt{d \log T})$-competitive algorithm. For simplicity, we consider the case where each body $K_{t}$ is a halfspace $\left\{x \mid a_{t}^{\top} x \geq b_{t}\right\}$. Henceforth, we will use $\mathbb{L}_{t}$ to denote the bit-complexity of the sequence $\left\{\left(a_{s}, b_{s}\right)\right\}_{s \leq t}$.

The basic idea is the following: (a) we show how to construct (weak) evaluation oracles for the work function $w_{t}$ and for the support function $h_{\Omega_{t}}$ of the body $\Omega_{t}$. Then (b) using Definition (2) and the evaluation oracle for the support function, we use random sampling to compute a point $x_{t}$ such that the expected error $\mathbb{E}\left[\left\|x_{t}-\operatorname{st}\left(\Omega_{t}\right)\right\|\right] \leq O\left(\frac{1}{t^{2}}\right) \cdot O P T$; this computation takes poly $\left(\mathbb{L}_{t}\right)$ time. Now the total expected error over all the timesteps is $O\left(\sum_{t} \frac{1}{t^{2}}\right) \cdot O P T=O(1) \cdot O P T$; this adds only a constant to the competitive ratio. Along the way, we need to change the algorithm slightly to control the bit-precision issues.

\subsection{The Modified Algorithm}

To ensure that $\Omega_{t}$ remains full-dimensional, we change the algorithm by stopping each phase when we know that $O P T$ has increased by a constant factor, but $\Omega_{t}$ still contains a large enough ball.

Note the changes in the algorithm: when the minimum work-function value (i.e., the optimal cost thus far) exceeds approximately $3 r / 2$, we reset our estimate $r$ for the work function. This ensures that (a) the optimal cost increases by a constant factor each time we reset $r$, and (b) when we reach line 6, the optimal value is guaranteed to be no more than $3 r / 2$, and hence $\Omega_{t}$ contains an $r / 2$ ball within it. The former property allows us to use the "doubling trick," and hence only the last phase matters. The latter is useful for the computations, since $\Omega_{t}$ is "centered" (it is sandwiched between two balls of $\Theta(r)$ radius).

There are two steps that need to be explained further. In Lemma 3.3 we show how to compute $\operatorname{MinWF}\left(w_{t}, \varepsilon\right)$, the minimum work function value up to additive error $\varepsilon$, which is needed in lines 3 and 4. In Section 3.3, we give a randomized algorithm to compute an approximate Steiner point of the body $\Omega_{t}$, needed in line 7 . This computation additionally requires the radius $R$ of a bounding ball for $\Omega$ (which we set to $2 r$ ), an error parameter (which we set to $r / t^{2}$ ), and a failure probability parameter $\delta$ (which we can set to 1 in our case since we are concerned only with expected cost). Lemma 3.5 then ensures that the expected distance from the true Steiner point is at most 


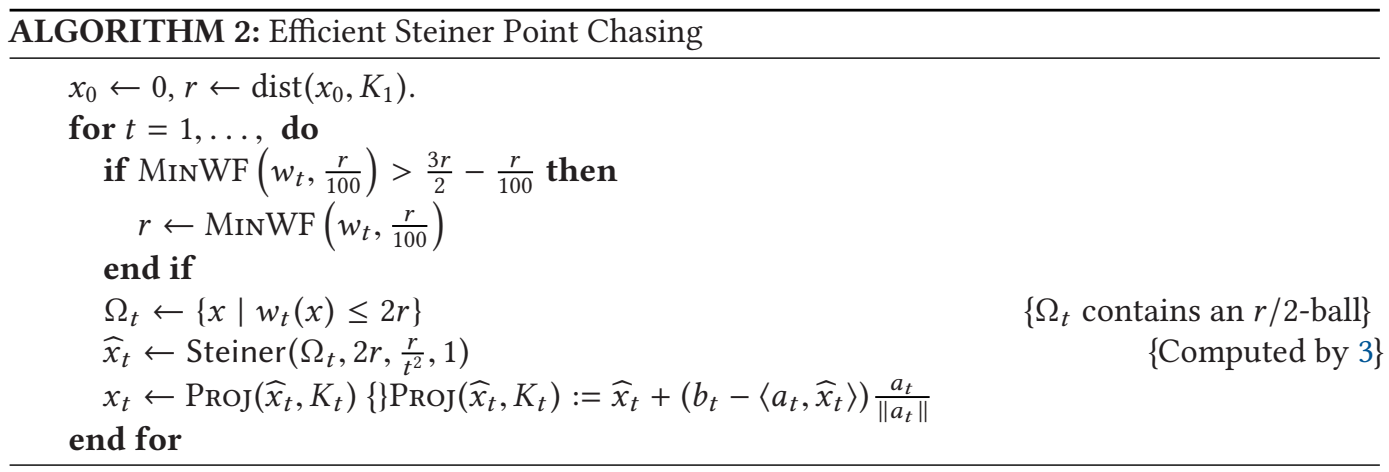

$\varepsilon_{t}=(1+\sqrt{\delta}) r / t^{2}=O\left(r / t^{2}\right)$. Finally, in line 8 we project this approximate Steiner point onto the halfspace to get a feasible point.

Lemma 3.1. Suppose the point $\widehat{x}_{t}$ satisfies $\mathbb{E}\left[\left\|\widehat{x}_{t}-s t\left(\Omega_{t}\right)\right\|\right] \leq \varepsilon_{t}$ and ALG outputs $x_{t}:=\Pi_{K_{t}}\left(\widehat{x}_{t}\right)$, the projection of $\widehat{x}_{t}$ onto the body $K_{t}$. Then the expected cost incurred by $A L G$ is at most $2 \sum \varepsilon_{t}$ greater than the algorithm that plays the Steiner point st $\left(\Omega_{t}\right)$ at each step.

Proof. Firstly, since $x_{t}$ is the projection of $\widehat{x}_{t}$ onto the body $K_{t}$, and $\operatorname{st}\left(\Omega_{t}\right) \in K_{t}$ by Lemma 2.2, we get $\left\|x_{t}-\operatorname{st}\left(\Omega_{t}\right)\right\| \leq\left\|\widehat{x}_{t}-\operatorname{st}\left(\Omega_{t}\right)\right\|$. Secondly, the triangle inequality implies

$$
\left\|x_{t}-x_{t-1}\right\| \leq\left\|x_{t}-\operatorname{st}\left(\Omega_{t}\right)\right\|+\left\|\operatorname{st}\left(\Omega_{t}\right)-\operatorname{st}\left(\Omega_{t-1}\right)\right\|+\left\|\operatorname{st}\left(\Omega_{t-1}\right)-x_{t-1}\right\| .
$$

Taking expectations, and using the assumptions of the lemma, we get

$$
\mathbb{E}\left[\left\|x_{t}-x_{t-1}\right\|\right] \leq \varepsilon_{t}+\left\|\operatorname{st}\left(\Omega_{t}\right)-\operatorname{st}\left(\Omega_{t-1}\right)\right\|+\varepsilon_{t-1} .
$$

Summing over all times completes the proof.

Corollary 3.2. The expected cost of Algorithm 2 is $O(\min (d, \sqrt{d \log T})) \cdot$ OPT. Furthermore, the algorithm runs in time poly $\left(\mathbb{L}_{T}\right)$.

Proof. The cost of the ideal algorithm that plays the actual Steiner point is $O(\min (d, \sqrt{d \log T}))$. $O P T$. Consider $r_{\text {final }}$, the final value of $r$ used by the algorithm; by construction, $r_{\text {final }}=O(1) \cdot O P T$. Since we chose $\varepsilon_{t} \leq O\left(1 / t^{2}\right) r_{\text {final }}$, the expected extra cost is $2 \sum_{t} \varepsilon_{t}=O(1) \cdot O P T$.

At step $t$, we can compute both MinWF and STEINER in poly $\left(\mathbb{L}_{t}, \log \left(1 / \varepsilon_{t}\right)\right)$. Note that $\mathbb{L}_{t} \leq \mathbb{L}_{T}$ and $\log \left(1 / \varepsilon_{T}\right)=\log (\operatorname{poly}(T)) \leq \operatorname{poly}\left(\mathbb{L}_{T}\right)$. The computation time for step $t$ is at most poly $\left(\mathbb{L}_{T}\right)$, and we perform at most $2 T$ such computations, hence the total computation time is bounded by $\operatorname{poly}\left(\mathbb{L}_{T}\right)$.

Next, we show how to compute the work function, and the Steiner point.

\subsection{Computing the Work and Support Functions}

In this section, we show how to compute the minimum value of the work function $w_{t}$ and the support function $h_{\Omega_{t}}$ of the body $\Omega_{t}$. The latter will be used in our estimation of the Steiner point in Section 3.3. We do each calculation by writing it as a second-order cone program.

Lemma 3.3 (Minimization of Work Function). Given a sequence $\left\{\left(a_{i}, b_{i}\right)\right\}_{i=1}^{t}$ and an error tolerance $\varepsilon>0$, there is a procedure MINWF $\left(w_{t}, \varepsilon\right)$ that outputs a value $v$ satisfying $\left|\min _{x} w_{t}(x)-v\right| \leq \varepsilon$ in time poly $\left(\mathbb{L}_{t}, \log (1 / \varepsilon)\right)$. 
Proof. Consider the following program on variables $\left(x_{1}, \lambda_{1}\right), \ldots,\left(x_{t}, \lambda_{t}\right)$, where each $\lambda_{i}$ is a scalar (and recall that $x_{0}=0$ ):

$$
\begin{array}{rlr} 
& \min \sum_{i=1}^{t} \lambda_{i} & \\
\text { s.t. }\left\|x_{i}-x_{i-1}\right\| \leq \lambda_{i} & & \forall i \in[t], \\
\left\langle a_{i}, x_{i}\right\rangle \geq b_{i} & & \forall i \in[t] .
\end{array}
$$

The above program is a second-order cone program. (Second-order cone programming is a special case of semidefinite programming.) By setting $x_{i}=\left(b_{i}+\delta\right) \frac{a_{i}}{\left\|a_{i}\right\|^{2}}$, we get that $\left\langle a_{i}, x_{i}\right\rangle \geq b_{i}+\delta$, and hence we get a point in the strict interior of the polyhedron $K_{1} \times K_{2} \times \ldots \times K_{t}$. Since there is a strictly feasible point, we know that the optimal primal and dual solutions exist, and have equal objective by [1, Thm 14]. Furthermore, we know that the optimal solution has bit-complexity bounded by poly $\left(\mathbb{L}_{t}\right)$. Therefore, we can solve this problem, e.g., using interior-point methods, to get an $\varepsilon$-approximate solution with runtime poly $\left(\mathbb{L}_{t}, \log (1 / \varepsilon)\right)(\operatorname{see}[5$, Section 4.6.2]).

Proposition 3.4 (Support Function Oracle). Given a sequence $\left\{\left(a_{i}, b_{i}\right)\right\}_{i=1}^{t}$ and a value $r>0$ (as defined in line 4), then we can implement an oracle $S_{U P \Omega_{\Omega_{t}}}$ for the support function $h_{\Omega_{t}}$ of $\Omega_{t}$ such that $\operatorname{SuPP}_{\Omega_{t}}(\theta, \varepsilon)$ outputs a value $v$ within $\varepsilon$ of $h_{\Omega_{t}}(\theta)$ and runs in time poly $\left(\mathbb{L}_{t}, \log \left(\frac{1}{\varepsilon}\right)\right)$.

Proof. We use a second-order cone program similar to the one in the previous proof. We add variables $x$ and $\lambda$ corresponding to an extra step after satisfying request $K_{t}$. Furthermore, we stipulate that the total distance traveled be at most $2 r$ to ensure that $x \in \Omega_{t}$.

$$
\begin{array}{rlrl}
\max \langle\theta, x\rangle & & \\
\text { s.t. }\left\|x_{i}-x_{i-1}\right\| & \leq \lambda_{i} & & \forall i \in[t], \\
\left\|x-x_{t}\right\| & \leq \lambda & & \forall i \in[t], \\
\left\langle a_{i}, x_{i}\right\rangle & \geq b_{i} & & \forall i \in[t], \\
\lambda+\sum_{i=1}^{t} \lambda_{i} & \leq 2 r . & &
\end{array}
$$

We will first show that the feasible set contains a point in the strict interior. Let $x_{t}^{*}$ denote the minimizer of the work function $w_{t}$, and let $\left(x_{1}^{*}, \ldots, x_{t}^{*}\right)$ be an optimal path witnessing the value of $w_{t}\left(x_{t}^{*}\right)$. We know that $\Omega_{t}$ contains the ball of radius $r / 2$ around $x_{t}^{*}$. Consider the points $x_{i}=x_{i}^{*}+\left(\frac{r}{10 \cdot t^{2}}\right) \frac{a_{i}}{\left\|a_{i}\right\|}$ and set $x=x_{t}$. By construction, we know that each $\left\langle a_{i}, x_{i}\right\rangle>b_{i}$. Furthermore, we get a point that satisfies the distance constraint strictly:

$$
\begin{aligned}
\sum_{i=1}^{t}\left\|x_{i}-x_{i-1}\right\|+\left\|x_{t}-x\right\| & =\sum_{i=1}^{t}\left\|x_{i}-x_{i-1}\right\| \\
& \leq \sum_{i=1}^{t}\left(\left\|x_{i}^{*}-x_{i-1}^{*}\right\|+2 \cdot \frac{r}{10 t^{2}}\right) \leq \frac{3 r}{2}+\sum_{i=1}^{t} \frac{r}{5 t^{2}}<2 r .
\end{aligned}
$$

The second inequality follows from the triangle inequality, and the third inequality comes from the early doubling in line 3. Since the optimal primal solution is bounded above by $2 r$ and there is a strictly feasible point, we know that the optimal primal and dual solutions exist, and have equal objective (again, by [1, Thm 14]). We can now use interior point methods to get an $\varepsilon$-approximate solution in time poly $\left(\mathbb{L}_{t}, \log \left(\frac{1}{\varepsilon}\right)\right)$ as in the preceding proof. 


\subsection{Approximating the Steiner Point}

Finally, we show how to approximate the Steiner point of a convex body using the oracle for the support function. Recall the definition (2) of the Steiner point $\operatorname{st}(K)$ as

$$
\operatorname{st}(K)=d \mathbb{E}_{\theta \sim S^{d-1}}\left[\theta h_{K}(\theta)\right],
$$

where $h_{K}(\theta):=\max _{x \in K}\langle\theta, x\rangle$ is the support function of the convex body $K$.

We are given halfspaces $\left\{\left(a_{1}, b_{1}\right), \ldots,\left(a_{t}, b_{t}\right)\right\}$ and access to an oracle $\operatorname{SupP}_{K}(\theta, \varepsilon)$ (defined in Proposition 3.4) to compute the support function; given a vector $\theta \in S^{d-1}$, this oracle returns a value $v$ such that $\left|v-h_{K}(\theta)\right| \leq \varepsilon$. We assume that we are also given a radius $R$ of a bounding ball $B(0, R) \supseteq K$. The estimation algorithm samples some number $N$ of random directions $\theta$, uses the oracle to get estimates for $h_{K}(\theta)$, and then uses the empirical mean as an estimate for the expectation in Equation (2). Formally, the algorithm is specified as Algorithm 3.

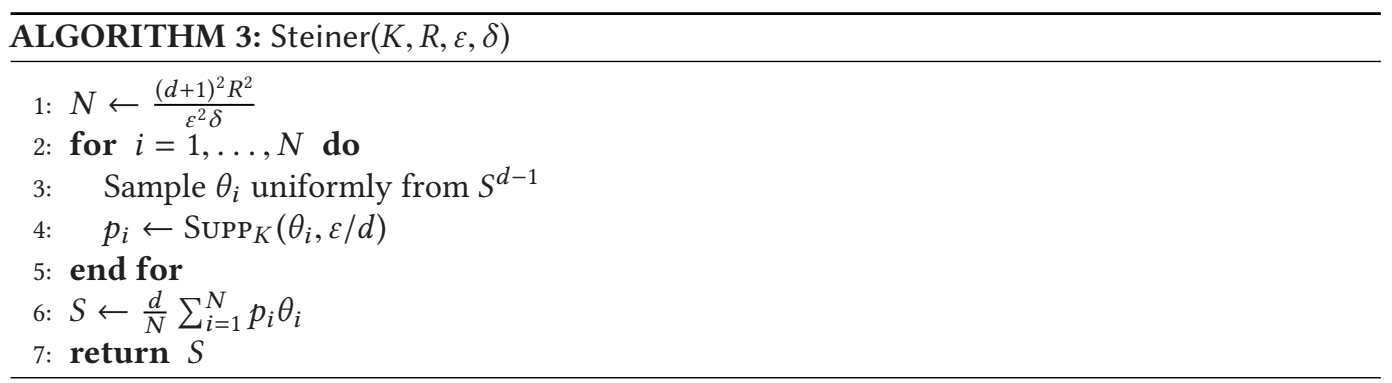

We show that Algorithm 3 computes a $2 \varepsilon$-approximate Steiner point both in expectation and with probability $1-\delta$, although only the former is necessary for this work.

Lemma 3.5. For $\varepsilon, \delta>0$, suppose $K \subseteq B(0, R)$ is a bounded convex body specified by a weak linear optimization oracle $\operatorname{SUPP}_{K}$. Let $\hat{x}$ be the output of Steiner $(K, R, \varepsilon, \delta)$. Then

(a) $\mathbb{E}[\|\hat{x}-s t(K)\|] \leq \varepsilon(1+\sqrt{\delta})$, and

(b) $\operatorname{Pr}[\|\hat{x}-s t(K)\| \leq 2 \varepsilon] \geq 1-\delta$.

Proof. Let $x:=\operatorname{st}(K)$. We first ignore the error of the linear optimization oracle and bound only the error due to sampling. Let $q_{i}:=h_{K}\left(\theta_{i}\right)$ be the true maximum value in the direction of the unit vector $\theta_{i}$ and let $\widehat{S}_{N}=\frac{d}{N} \sum_{i=1}^{N} q_{i} \theta_{i}$. Since the $\left(\theta_{i}, q_{i}\right)$ are i.i.d. samples with $\mathbb{E}\left[d q_{i} \theta_{i}\right]=\operatorname{st}(K)=x$ and $q_{i} \leq \max _{z \in B(0, R)}\left\langle\theta_{i}, z\right\rangle=R$, we have

$$
\mathbb{E}\left[\left\|\widehat{S}_{N}-\operatorname{st}(K)\right\|^{2}\right]=\mathbb{E}\left[\left\|\frac{1}{N} \sum_{i=1}^{N}\left(d q_{i} \theta_{i}-x\right)\right\|^{2}\right]=\frac{1}{N^{2}} \sum_{i=1}^{N} \mathbb{E}\left[\left\|d q_{i} \theta_{i}-x\right\|^{2}\right] \leq \frac{(d+1)^{2} R^{2}}{N} \leq \varepsilon^{2} \delta .
$$

Now Jensen's inequality says $\mathbb{E}\left[\left\|\widehat{S}_{N}-\operatorname{st}(K)\right\|\right]^{2} \leq \mathbb{E}\left[\left\|\widehat{S}_{N}-\operatorname{st}(K)\right\|^{2}\right] \leq \varepsilon^{2} \delta$. The second-to-last inequality follows since both $q_{i} \theta_{i}$ and $x$ lie in $B(0, R)$. Moreover, using Markov's inequality in conjunction with the second inequality also implies that $\left\|\widehat{S}_{N}-\operatorname{st}(K)\right\| \leq \varepsilon$ with probability at least $1-\delta$.

Finally, to account for error in the weak linear optimization oracle, note that the magnitude of each error is $\left|p_{i}-q_{i}\right|<\varepsilon / d$. Hence, the convexity of norms implies that the additional error between the algorithm's output $S$ and the true estimate $\widehat{S}_{N}$ is $\left\|S-\widehat{S}_{N}\right\| \leq \varepsilon$. The triangle inequality completes the proof. 


\section{ACKNOWLEDGMENTS}

We thank Nikhil Bansal, Sébastien Bubeck, Niv Buchbinder, Marco Molinaro, Seffi Naor, Kirk Pruhs, and Cliff Stein for comments and discussions.

\section{REFERENCES}

[1] Farid Alizadeh and Donald Goldfarb. 2003. Second-order cone programming. Mathematical Programming 95, 1 (2003), 3-51.

[2] Antonios Antoniadis, Neal Barcelo, Michael Nugent, Kirk Pruhs, Kevin Schewior, and Michele Scquizzato. 2016. Chasing convex bodies and functions. In LATIN. Springer, Berlin, 68-81. https://doi.org/10.1007/978-3-662-49529-2_6

[3] C. J. Argue, Sébastien Bubeck, Michael B. Cohen, Anupam Gupta, and Yin Tat Lee. 2019. A nearly-linear bound for chasing nested convex bodies. In SODA. SIAM, 117-122.

[4] Nikhil Bansal, Martin Böhm, Marek Eliáš, Grigorios Koumoutsos, and Seeun William Umboh. 2018. Nested convex bodies are chaseable. SODA (2018).

[5] Ahron Ben-Tal and Arkadi Nemirovski. 2001. Lectures on Modern Convex Optimization: Analysis, Algorithms, and Engineering Applications. Vol. 2. SIAM.

[6] Yoav Benyamini and Joram Lindenstrauss. 2000. Geometric Nonlinear Functional Analysis. Vol. 1. AMS Colloquium Publications, Vol. 48. American Mathematical Society, Providence, RI. xii+488 pages.

[7] Allan Borodin, Nathan Linial, and Michael E. Saks. 1992. An optimal on-line algorithm for metrical task system. F. Assoc. Comput. Mach. 39, 4 (1992), 745-763. https://doi.org/10.1145/146585.146588

[8] Sébastien Bubeck, Bo'az Klartag, Yin Tat Lee, Yuanzhi Li, and Mark Sellke. 2018. Chasing nested convex bodies nearly optimally. CoRR abs/1811.00999 (2018). arXiv:1811.00999. http://arxiv.org/abs/1811.00999.

[9] Sébastien Bubeck, Yin Tat Lee, Yuanzhi Li, and Mark Sellke. 2019. Competitively chasing convex bodies. In Proceedings of the 51st Annual ACM SIGACT Symposium on Theory of Computing (STOC'19). 861-868. https://doi.org/10.1145/ 3313276.3316314

[10] Niangjun Chen, Gautam Goel, and Adam Wierman. 2018. Smoothed online convex optimization in high dimensions via online balanced descent. arXiv:1803.10366.

[11] Joel Friedman and Nathan Linial. 1993. On convex body chasing. Discrete Comput. Geom. 9, 3 (1993), 293-321. https: //doi.org/10.1007/BF02189324

[12] Hiroshi Fujiwara, Kazuo Iwama, and Kouki Yonezawa. 2008. Online chasing problems for regular polygons. Inform. Process. Lett. 108, 3 (2008), 155-159.

[13] Gautam Goel, Yiheng Lin, Haoyuan Sun, and Adam Wierman. 2019. Beyond online balanced descent: An optimal algorithm for smoothed online optimization. arXiv:1905.12776.

[14] Elias Koutsoupias and Christos H. Papadimitriou. 1995. On the k-server conjecture. J. ACM (JACM) 42, 5 (1995), 971-983.

[15] Krzysztof Przesławski. 1996. Centres of convex sets in $L^{p}$ metrics. J. Approx. Theory 85, 3 (1996), 288-296. https: //doi.org/10.1006/jath.1996.0043

[16] Rolf Schneider. 2014. Convex Bodies: The Brunn-Minkowski Theory. Encyclopedia of Mathematics and its Applications, Vol. 151. Cambridge University Press, Cambridge. xxii+736 pages.

[17] Mark Sellke. 2019. Chasing convex bodies optimally. CoRR abs/1905.11968 (2019). arXiv:1905.11968. http://arxiv.org/ abs/1905.11968.

[18] René Sitters. 2014. The generalized work function algorithm is competitive for the generalized 2-server problem. SIAM f. Comput. 43, 1 (2014), 96-125. https://doi.org/10.1137/120885309

Received February 2020; revised February 2021; accepted February 2021 